\title{
Production of ordered silicon nanocrystals by low-energy ion sputtering
}

\author{
Raúl Gago and Luis Vázquez ${ }^{\mathrm{a})}$ \\ Instituto de Ciencia de Materiales de Madrid (CSIC), Cantoblanco, 28049 Madrid, Spain \\ Rodolfo Cuerno \\ Departamento de Matemáticas \& GISC, Universidad Carlos III de Madrid, Avenida Universidad 30, 28911 \\ Leganés, Spain \\ María Varela and Carmen Ballesteros \\ Departamento de Física, Universidad Carlos III de Madrid, Avenida Universidad 30, 28911 Leganés, Spain \\ José María Albella \\ Instituto de Ciencia de Materiales de Madrid (CSIC), Cantoblanco, 28049 Madrid, Spain
}

(Received 19 January 2001; accepted for publication 23 March 2001)

\begin{abstract}
We report on the production of ordered assemblies of silicon nanostructures by means of irradiation of a $\mathrm{Si}(100)$ substrate with $1.2 \mathrm{keV} \mathrm{Ar}{ }^{+}$ions at normal incidence. Atomic force and high-resolution transmission electron microscopies show that the silicon structures are crystalline, display homogeneous height, and spontaneously arrange into short-range hexagonal ordering. Under prolonged irradiation (up to $16 \mathrm{~h}$ ) all dot characteristics remain largely unchanged and a small corrugation develops at long wavelengths. We interpret the formation of the dots as a result of an instability due to the sputtering yield dependence on the local surface curvature. (C) 2001 American Institute of Physics. [DOI: 10.1063/1.1372358]
\end{abstract}

The production of semiconductor nanostructures has attracted the interest of many research groups because of the important applications in optoelectronic and quantum devices. ${ }^{1}$ Many of the interesting materials properties depend on the size, shape, and regularity of the nanometric substructure. In particular, efficient light emission from silicon is achieved when the nanostructures are smaller than the bulk exciton. ${ }^{2,3}$ Silicon nanostructures are very important for the development of new types of microelectronic, electrooptical, electrochemical, electromechanical, sensing, and silicon laser devices. ${ }^{3-5}$ Techniques such as Si ion implantation, electron-beam writing, scanning probe litography, pulsed laser deposition, laser annealing, low pressure chemical vapor deposition, and thermal evaporation have been used to fabricate silicon nanostructures. ${ }^{3,6}$ However, it is not evident that any of these techniques can be used to simultaneously control purity, uniformity, and crystallinity of the nanostructures and to produce these efficiently in a large scale. Thus, processes of fabrication of silicon nanocrystals are being investigated. ${ }^{4,7}$ In this sense both the ease and reproducibility of the process involved are key factors for its eventual practical use. One very promising candidate is irradiation of a monocrystalline semiconductor surface by low energy ions. This technique has already proved its capability for the production of self-organized quantum dots on $\mathrm{GaSb}^{8}{ }^{8}$ and InP surfaces, ${ }^{9}$ with promising photoluminescence properties. ${ }^{8}$ However, although the production of ripple structures on silicon surfaces has been reported, ${ }^{10}$ no report exists on the application of this irradiation technique to produce nanocrystalline dots on silicon surfaces.

In this letter we report on the production and morphological characterization of nanocrystalline silicon dots on $\mathrm{Si}$

${ }^{a)}$ Electronic mail: 1vb@icmm.csic.es
(100) surfaces by $1.2 \mathrm{keV} \mathrm{Ar}^{+}$ion bombardment. We have produced these nanostructures over a wide temporal range spanning from 2 min up to $16 \mathrm{~h}$. These experiments have allowed us to confirm the morphological stability of the features produced, as well as to observe the development of a long wavelength corrugation. As a result we interpret the physical mechanism determining the surface morphology to be an instability due to the local surface curvature dependence of the sputtering yield. ${ }^{11,12}$

The samples were processed in a high vacuum chamber with a base pressure of $2 \times 10^{-7}$ mbar. Si (100) wafers were placed on the sampleholder and irradiated with an $\mathrm{Ar}^{+}$beam from a $3 \mathrm{~cm}$ Kauffman ion gun. Argon ions, accelerated at $1.2 \mathrm{keV}$, impinged normally on the silicon surface with an effective ion current density of $0.24 \mathrm{~mA} / \mathrm{cm}^{2}$. The irradiation time was varied between $1 \mathrm{~min}$ and $16 \mathrm{~h}$. The bombarded samples were studied by atomic force microscopy (AFM) and high-resolution transmission electron microscopy (HRTEM). AFM measurements were performed in air in tapping mode, using silicon cantilevers (10 $\mathrm{nm}$ radius). Specimens suitable for HRTEM were prepared by standard procedures and examined using a Philips CM200 FEG analytical microscope operating at $200 \mathrm{kV}$.

Figure 1 shows AFM images of samples bombarded for 6 min [panel (a)] and 960 min [panel (b)]. Silicon dots, 6 $\pm 1 \mathrm{~nm}$ high and $40-50 \mathrm{~nm}$ of diameter are clearly seen. The AFM images suggest two effects: first, the nearest neighbor distance between dots tends to be a time independent constant; second, the nanostructures self-organize into shortrange hexagonal symmetry, even after $16 \mathrm{~h}$ of sputtering. These two facts, which imply a constant dot surface density of $\approx 3.5 \times 10^{10} \mathrm{dots} / \mathrm{cm}^{2}$, are confirmed in panels (c) and (d) of Fig. 1, where the two-dimensional autocorrelations of the AFM images show short-range hexagonal ordering with a 

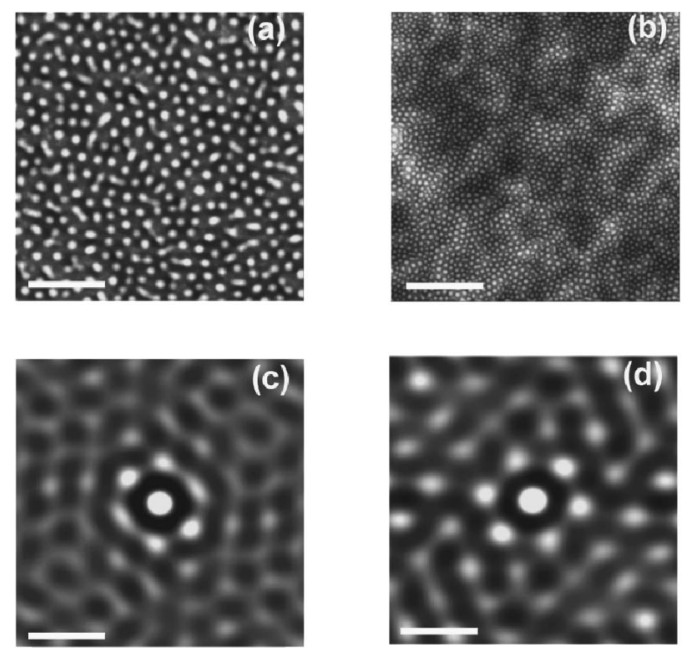

FIG. 1. AFM images of a $\mathrm{Si}(100)$ substrate sputtered by $1.2 \mathrm{keV} \mathrm{Ar}^{+}$ions at normal incidence for $6 \mathrm{~min}\left[(\mathrm{a}): 1 \times 1 \mu \mathrm{m}^{2}\right]$ and $960 \mathrm{~min}$ [(b): 3 $\times 3 \mu \mathrm{m}^{2}$ ]. (c) and (d): Two-dimensional autocorrelation functions obtained from $400 \times 400 \mathrm{~nm}^{2}$ areas of images (a) and (b), respectively. The bars represent $277 \mathrm{~nm}$ [panel (a)], $831 \mathrm{~nm}$ [panel (b)], and $111 \mathrm{~nm}$ [panels (c) and (d)].

nearest dot distance of $\approx 55 \mathrm{~nm}$. As seen in Fig. 1(b), after 960 min of irradiation, the dot structure is clear but the surface also displays a long wavelength corrugation, $\approx 500 \mathrm{~nm}$ wide and $\approx 6 \mathrm{~nm}$ high.

An important issue concerning the potential application of these dot structures is their crystallinity. ${ }^{4,5} \mathrm{~A}$ crosssectional multibeam TEM image of a 10 min sputtered sample along the $\langle 110\rangle$ direction is shown in Fig. 2. The formation of uniformly distributed nanostructures with lenticular shape covered by an amorphous layer $\approx 2 \mathrm{~nm}$ thick is observed. The inset of Fig. 2 shows a high-resolution image of one of the nanostructures on the main panel. $\{111\}$ lattice fringes are visible, showing the crystallinity of the nanostructures and a low number of defects. The height of the nanocrystals, that is excluding the amorphous layer, is in the range $6-7.5 \mathrm{~nm}$, its width being in the range $40-60 \mathrm{~nm}$. These figures are in agreement with those obtained by AFM provided that tip convolution effects are considered. The silicon dots shape and size induced by ion sputtering are different from those observed for $\mathrm{GaSb},{ }^{8}$ which are $30 \times 30 \mathrm{~nm}$ conical

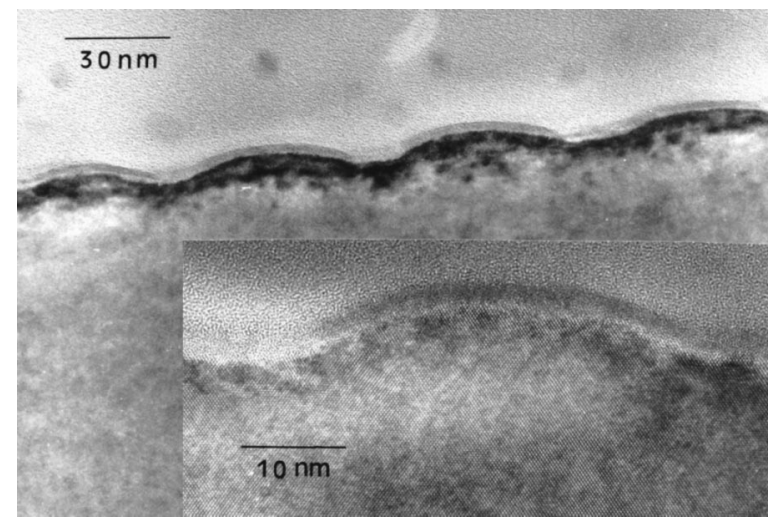

FIG. 2. Cross-sectional HRTEM multibeam image along the $\langle 110\rangle$ direction of a sputtered sample. $\mathrm{Ar}^{+}$ions at $1.2 \mathrm{keV}$ were employed at normal incidence for $10 \mathrm{~min}$. Inset: high-resolution image of one of the nanostructures on the main panel. $\{111\}$ lattice fringes show the crystallinity of the nano-

structures; a low number of defects are visible.
Downloaded 24 Feb 2010 to 161.111 .180 .191 . Redistribution subject to AlP license or copyright; see http://apl.aip.org/apl/copyright.jsp

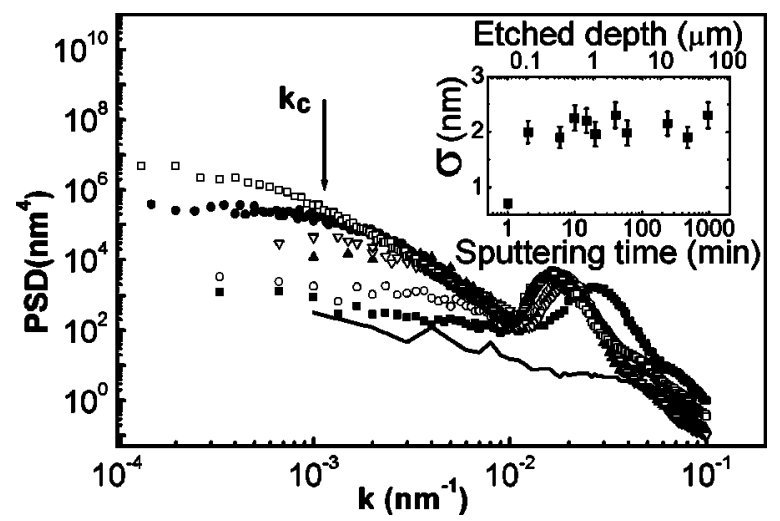

FIG. 3. Log-log plot ot the PSD curves obtained from AFM images of Si (100) substrates sputtered by $1.2 \mathrm{keV} \mathrm{Ar}^{+}$ions at normal incidence for 1 is indicated for the sample irradiated for $960 \mathrm{~min}$. Inset: surface roughness $\sigma$ vs sputtering time and etched depth. 1 min sputtering time amounts to an ion dose equal to $9 \times 10^{16} \mathrm{~cm}^{-2}$

dots. We note that for the GaSb system a preferential sputtering of the $\mathrm{Sb}$ atoms was observed, which is not the case for our system. This fact could be related to the different dot morphologies observed for both cases.

In order to obtain a better insight into the mechanism of dot formation for silicon we have prepared samples for times ranging from $1 \mathrm{~min}$ up to $16 \mathrm{~h}$. The samples were systematically analyzed by AFM. The morphological data were quantitatively analyzed within the framework of the dynamic scaling theory. ${ }^{13,14}$ An important magnitude is the root mean square roughness of the surface, $\sigma$. The dynamic scaling theory predicts that a typical lateral length scale $\xi$ of the system grows as $\xi \sim t^{1 / z}$, where $t$ is the sputtering time and $1 / z$ is a coarsening exponent. As a consequence, when probing the surface over a distance $L>t^{1 / z}$, the roughness grows as $\sigma \sim t^{\beta}$, whereas for $t^{1 / z}>L$ the roughness saturates to $\sigma$ $\sim L^{\alpha}$. Thus, the exponents $\alpha$ and $z$ describe the interface evolution. The ratio $\beta \equiv \alpha / z$ can be obtained directly by plotting the time evolution of the roughness $\sigma$, as measured by AFM; moreover, $\alpha$ can be obtained from the analysis of the power spectral density (PSD) of the surface morphology measured by AFM, since the PSD behaves, for a twodimensional rough surface, ${ }^{14}$ as $\operatorname{PSD}(k) \sim 1 / k^{2 \alpha+2}$, with $k$ $=1 / L$. Figure 3 is a logarithmic plot of the PSD of the surface morphology measured for 1, 2, 6, 20, 60, 240, and 960 min of sputtering. The large peak in the PSDs corresponds to the average distance between nearest neighbor dots. We observe that this wavelength increases with time from $\approx 35 \mathrm{~nm}$ for 2 min until it reaches a value in the 52-60 $\mathrm{nm}$ range for times longer than $6 \mathrm{~min}$. For sputtering times longer than 20 min the PSD displays for small $k$ values an exponent value $\alpha=0.8 \pm 0.1$. For each time there is a minimum wavelength $k_{c}$ below which the PSD crosses over to a different behavior. We obtain that $k_{c}$ decreases as $k_{c} \sim t^{-0.2}$. Hence, length scales larger than the nearest dot distance and smaller than $1 / k_{c}$ display scale invariant behavior with exponents $\alpha$ $\simeq 0.8$ and $1 / z \simeq 0.2$. This corresponds to the development of a long wavelength corrugated structure, which appears after 20 min of sputtering and coarsens with time, see Fig. 1(b).

Finally, the inset of Fig. 3 shows the change of $\sigma$ with the sputtering time. The roughness increases sharply during AIP license or copyright; see http://apl.aip.org/apl/copyright.jsp (solid line), $2(\mathbf{\square}), 6(\bigcirc), 20(\mathbf{\Delta}), 60(\nabla), 240(\bullet)$, and $960(\square)$ min. $k_{c}$ 
the very first stages of the sputtering process, but rapidly attains a constant value $\approx 2 \mathrm{~nm}$. This extremely small roughness is obtained even after a 50- $\mu \mathrm{m}$ thick layer of the silicon wafer has been etched away by the $\mathrm{Ar}^{+}$ions. We have found that for long sputtering times the main morphological difference is the development of the long wavelength corrugation. However, the PSD curves (Fig. 3) indicate that the main contribution to the total surface roughness $\sigma$ is due to the dot nanostructures, rather than to the long wavelength corrugation.

The observed behavior is in qualitative agreement with the picture of the sputtering process based on the interplay between roughening induced by ion sputtering and smoothening due to surface diffusion. ${ }^{11,12,15,16}$ The instability due to the local surface curvature dependence of the sputtering yield ${ }^{11}$ produces at short times a regular array of dots (with defects induced by shot noise in the ion beam ${ }^{12}$ ) and a rapid increase of the roughness. A similar qualitative behavior has been reported for the GaSb and InP dots. ${ }^{8,9}$ At longer times, there are nonlinear effects ${ }^{12}$ inducing drastically slower growth for the roughness ${ }^{15,16}$ as well as scaling behavior at length scales larger than the nearest dot distance, of the type encountered for the long wavelength corrugation [Fig. 1(b)]. In Ref. 16, the production of either dots or holes by ion sputtering has been predicted to depend on the ion penetration depth and the shape of the collision cascade. Our experimental results agree quite well with the overall scenario thus predicted for the case of a positive nonlinearity. It will be interesting to test ${ }^{17}$ further predictions ${ }^{16}$ on morphology behavior as a function of temperature and ion energy.

In summary, we have shown that crystalline dots $(\approx 7 \mathrm{~nm}$ high and $\approx 50 \mathrm{~nm}$ wide) are produced on a $\mathrm{Si}(100)$ surface by low energy $\mathrm{Ar}^{+}$ion bombardment at normal incidence. The silicon nanocrystals arrange themselves into hexagonal short-range ordering from 2 up to $960 \mathrm{~min}$ of sputtering. The main effect of prolonged sputtering is the development of a long wavelength corrugation displaying scaling properties, which does not largely contribute to the total surface roughness. The experimental observations as well as the dynamic scaling analysis of the AFM images are consistent with the formation of the dots as a result of an instability due to the curvature dependence of the sputtering yield.

This work has been partially supported by DGES (Spain) Grant Nos. MAT1999-0830-C03-01, MAT2000-0375-C0202, and BFM2000-0006.

${ }^{1}$ G. Schmidt and L. F. Chi, Adv. Mater. 10, 515 (1998).

${ }^{2}$ E. Chomski and G. A. Ozin, Adv. Mater. 12, 1071 (2000).

${ }^{3}$ L. Pavesi, L. Dal Negro, C. Mazzoleni, G. Franz, and F. Priolo, Nature (London) 408, 440 (2000).

${ }^{4}$ Y. Yin, B. Gates, and Y. Xia, Adv. Mater. 12, 1426 (2000).

${ }^{5}$ G. F. Grom, D. J. Lockwood, J. P. McCaffrey, H. J. Labbé, P. M. Fauchet, B. White, Jr., J. Diener, D. Kovalev, F. Koch, and L. Tsybeskov, Nature (London) 407, 358 (2000).

${ }^{6}$ S. Hu, A. Hamidi, S. Altemeyer, T. Koster, B. Spangenberg, and H. Kurz, J. Vac. Sci. Technol. B 16, 2822 (1998); J. W. Lyding, T. C. Shen, J. S. Hubacek, J. R. Tucker, and G. C. Abein, Appl. Phys. Lett. 64, 2010 (1994); N. Suzuki, T. Makino, Y. Yamada, T. Yoshida, and S. Onari, ibid. 76, 1309 (2000); T. Toyama, Y. Kotani, A. Shimode, and H. Okamoto, ibid. 73, 105 (1998); E. Edelberg, S. Bergh, R. Wame, M. Hall, and E. S. Aydil, ibid. 68, 1415 (1997); E. I. Givargizov, J. Vac. Sci. Technol. B 11, 449 (1993); A. M. Morales and C. M. Lieber, Science 279, 208 (1998).

${ }^{7}$ N. Miyata, H. Watanabe, and M. Ichikawa, Appl. Phys. Lett. 77, 1620 (2000).

${ }^{8}$ S. Facsko, T. Dekorsy, C. Koerdt, C. Trappe, H. Kurz, A. Vogt, and H. L. Hartnagel, Science 285, 1551 (1999).

${ }^{9}$ F. Frost, A. Schindler, and F. Bigl, Phys. Rev. Lett. 85, 4116 (2000).

${ }^{10}$ G. Carter and V. Vishnyakov, Phys. Rev. B 54, 17647 (1996).

${ }^{11}$ R. M. Bradley and J. M. E. Harper, J. Vac. Sci. Technol. A 6, 2390 (1988).

${ }^{12}$ R. Cuerno and A.-L. Barabási, Phys. Rev. Lett. 74, 4746 (1995).

${ }^{13}$ F. Family and T. Vicsek, J. Phys. A 18, L15 (1985).

${ }^{14}$ A.-L. Barabási and H. E. Stanley, Fractal Concepts in Surface Growth (Cambridge University Press, Cambridge, 1995).

${ }^{15}$ S. Park, B. Kahng, H. Jeong, and A.-L. Barabási, Phys. Rev. Lett. 83, 3486 (1999).

${ }^{16}$ B. Kahng, H. Jeong, and A.-L. Barabási, Appl. Phys. Lett. 78, 805 (2001).

${ }^{17}$ R. Gago, L. Vázquez, R. Cuerno, M. Varela, C. Ballesteros, and J. M. Albella (unpublished). 